\title{
T helper 2 and regulatory T-cell cytokine production by mast cells: a key factor in the pathogenesis of IgG4-related disease
}

Mai Takeuchi1,6, Yasuharu Sato ${ }^{1,6}$, Kyotaro Ohno ${ }^{1}$, Satoshi Tanaka ${ }^{2}$, Katsuyoshi Takata ${ }^{1}$, Yuka Gion ${ }^{1}$, Yorihisa Orita ${ }^{3}$, Toshihiro Ito ${ }^{4}$, Tomoyasu Tachibana ${ }^{5}$ and Tadashi Yoshino ${ }^{1}$

${ }^{1}$ Department of Pathology, Okayama University Graduate School of Medicine, Dentistry and Pharmaceutical Sciences, Okayama, Japan; ${ }^{2}$ Department of Immunochemistry, Okayama University Graduate School of Medicine, Dentistry and Pharmaceutical Sciences, Okayama, Japan; ${ }^{3}$ Department of Otolaryngology, Head and Neck Surgery, Okayama University Graduate School of Medicine, Dentistry and Pharmaceutical Sciences, Okayama, Japan; ${ }^{4}$ Department of Pathology and Experimental Medicine, Okayama University Graduate School of Medicine, Dentistry and Pharmaceutical Sciences, Okayama, Japan and ${ }^{5}$ Department of Otolaryngology, Himeji Red Cross Hospital, Himeji, Japan

IgG4-related disease is a systemic disorder with unique clinicopathological features and uncertain etiological features and is frequently related to allergic disease. T helper 2 and regulatory T-cell cytokines have been reported to be upregulated in the affected tissues; thus, the production of these cytokines by $T$ helper 2 and regulatory $\mathrm{T}$ cells has been suggested as an important factor in the pathogenesis of IgG4-related disease. However, it is not yet clear which cells produce these cytokines in IgG4-related disease, and some aspects of the disorder cannot be completely explained by T-cell-related processes. To address this, we analyzed paraffinembedded sections of tissues from nine cases of IgG4-related submandibular gland disease, five cases of submandibular sialolithiasis, and six cases of normal submandibular gland in order to identify potential key players in the pathogenesis of IgG4-related disease. Real-time polymerase chain reaction analysis confirmed the significant upregulation of interleukin (IL)4, IL10, and transforming growth factor beta 1 (TGF $\beta 1$ ) in IgG4related disease. Interestingly, immunohistochemical studies indicated the presence of mast cells expressing these cytokines in diseased tissues. In addition, dual immunofluorescence assays identified cells that were double-positive for each cytokine and for KIT, which is expressed by mast cells. In contrast, the distribution of $T$ cells did not correlate with cytokine distribution in affected tissues. We also found that the mast cells were strongly positive for IgE. This observation supports the hypothesis that mast cells are involved in IgG4-related disease, as mast cells are known to be closely related to allergic reactions and are activated in the presence of elevated non-specific IgE levels. In conclusion, our results indicate that mast cells produce $T$ helper 2 and regulatory T-cell cytokines in tissues affected by IgG4-related disease and possibly have an important role in disease pathogenesis.

Modern Pathology (2014) 27, 1126-1136; doi:10.1038/modpathol.2013.236; published online 3 January 2014

Keywords: cytokine; IgE; IgG4-related disease; mast cells; regulatory T cell

IgG4-related disease has recently been recognized as a clinical entity with unique clinicopathological features that can affect systemic organs. ${ }^{1-4}$

Correspondence: Dr Y Sato, Department of Pathology, Okayama University Graduate School of Medicine, Dentistry and Pharmaceutical Sciences, 2-5-1, Shikata-cho, Kita-ku, Okayama 700-8558, Japan.

E-mail: satou-y@cc.okayama-u.ac.jp

${ }^{6}$ These authors contributed equally to this work.

Received 31 July 2013; revised 3 November 2013; accepted 4 November 2013; published online 3 January 2014
Histological examination of IgG4-related disease has shown diffuse lymphoplasmacytic infiltration, interstitial fibrosis, obliterative phlebitis, and eosinophilic infiltration. Additionally, numerous IgG4positive plasma cells are observed and the IgG4positive/IgG-positive cell ratio is elevated above $40 \%$ in affected tissues. It has been suggested that these distinctive features are caused by the $\mathrm{T}$ helper 2 cell cytokines interleukin (IL) 4 and IL5 and the regulatory T-cell cytokines IL10 and transforming growth factor beta 1 (TGF $\beta 1$ ). ${ }^{5,6}$ These cytokines are 
upregulated in the tissues affected by IgG4-related disease, where IL4 and IL10 are thought to stimulate $\mathrm{B}$ cells and plasma cells to induce lymphoplasmacytic infiltration and IgE and IgG4 production, whereas TGF $\beta 1$ is thought to induce interstitial fibrosis. On the basis of these observations, T helper 2 and regulatory $\mathrm{T}$ cells have been considered to have a key role in the pathogenesis of IgG4-related disease. However, it has not been definitively determined whether these $\mathrm{T}$ cells are actually responsible for the production of such cytokines in affected tissues. Moreover, the hypothesis that $\mathrm{T}$ cells produce the disease-related cytokines does not explain why an anti-CD20 monoclonal antibody, rituximab, would be effective in treating refractory IgG4-related disease patients. ${ }^{7}$

An alternative to the T-cell hypothesis is the involvement of mast cells in IgG4-related disease. Mast cells were first found to release histamine granules in immediate hypersensitivity reactions; however, recent research has revealed that mast cells are involved in a variety of immune responses, including host defense, immune regulation, allergy, chronic inflammation, and autoimmune disease. ${ }^{8}$ In response to IgE stimulation, mast cells secrete various mediators, including $\mathrm{T}$ helper 2 cytokines and regulatory cytokines. ${ }^{9}$ Non-specific IgE alone can induce cytokine secretion independent of any antigen. ${ }^{10}$ As IgG4-related disease is frequently complicated with allergic disease and accompanied by elevation of serum IgE levels, we hypothesized that mast cells may be involved in the pathogenesis of IgG4-related disease.

\section{Materials and methods}

\section{Samples}

Tissue samples from nine cases of submandibular gland IgG4-related disease were obtained. The serum IgG4 levels were elevated in all nine cases. Samples from five cases of submandibular sialolithiasis and six cases of normal submandibular glands which were resected during treatment for oral cancer were obtained and used as disease controls. Formalinfixed paraffin-embedded specimens were used for immunohistochemistry, dual immunofluorescence, RNA extraction, and real-time polymerase chain reaction (PCR) analysis. All samples were obtained with the approval of the Institutional Review Board at Okayama University.

\section{Real-Time Quantitative PCR}

Total RNA was extracted from the paraffin-embedded sections of all samples by using an miRNeasy FFPE Kit (QIAGEN, Valencia, CA, USA). Complementary DNA was prepared by reverse transcription PCR by using a SuperScript VILO MasterMix kit (Invitrogen, Carlsbad, CA, USA). Multiplex real-time PCR was performed for quantitative analysis, according to the standard protocol by using Taqman Gene Expression Assays (Applied Biosystems, Foster City, CA, USA) and a Step One Plus Real-Time PCR System (Applied Biosystems). Specific primers and probes for TGF $\beta 1$, IL4, IL5, IL10, and $\beta$-actin were obtained from Applied Biosystems. The PCR cycling conditions were as follows: $30 \mathrm{~s}$ at $95^{\circ} \mathrm{C}$ and 50 cycles of $5 \mathrm{~s}$ at $95{ }^{\circ} \mathrm{C}$, and $30 \mathrm{~s}$ at $60^{\circ} \mathrm{C}$. The expression of each cytokine was normalized to that of $\beta$-actin, which was used as an endogenous control.

\section{Histological Examination and Immunohistochemistry}

All of the diseased and normal tissue samples used in this study were surgically resected specimens of submandibular glands. The specimens were fixed in $10 \%$ formaldehyde and embedded in paraffin. Serial $4-\mu$ m-thick sections were cut from the block of paraffin-embedded tissue and stained with hematoxylin and eosin (H\&E). The sections were immunohistochemically stained using an automated Bond Max stainer (Leica Biosystems, Melbourne, Germany). The following primary antibodies were used: TGF $\beta 1$ (ab49754; 1:100; Novocastra, Newcastle, UK), IL4 (orb22602; 1:400; Biorbit, Cambridge, UK), IL5 (MAB605; 1:400; R\&D, Minneapolis, MN, USA), IL10 (orb22606; 1:100; Biorbit), KIT/CD117 (YR145; 1:100; EPITOMICS, Burlingame, CA, USA), IgG (polyclonal; 1:20 000; Dako, Glostrup, Denmark), IgG4 (HP6025; 1:10000; The Binding Site, Birmingham, UK), forkhead box P3 (FOXP3) (236A/E7; 1:100; Abcam, Cambridge, UK), CD4 (1F6; 1:40; Nichirei, Tokyo, Japan), and IgE (A094; 1:500; Dako).

Following immunostaining, the number of IgG4positive and IgG-positive cells was estimated in areas with the highest density of IgG4-positive cells. In accordance with the consensus statement on the pathological features of IgG4-related disease published in 2012, ${ }^{2}$ three different high-power fields $(\mathrm{HPFs})$ (eyepiece, $\times 10$; lens, $\times 40$ ) were examined to calculate the average number of IgG4-positive cells per HPFs and the IgG4-positive/IgG-positive cell ratio. Cells that were positive for each cytokine, KIT, FOXP3, and IgE were counted in the three different fields (eyepiece, $\times 10$; lens, $\times 20$ ) determined to have the highest density of positive cells. The average number of positive cells per square millimeter $\left(\mathrm{mm}^{2}\right)$ was calculated.

\section{Dual Immunofluorescence Assays}

For indirect dual immunofluorescence assays, paraffin sections were stained with the primary antibodies for KIT and TGF $\beta 1$, KIT and IL4, KIT and IL5, or KIT and IL10. Fluorescein isothiocyanate (FITC)-conjugated secondary antibodies (Alexa Fluor anti-mouse 555 and Alexa Fluor anti-rabbit 488; both Invitrogen Co, Carlsbad, CA, USA) were used at a dilution of 1:400. The stained specimens 
were examined with a conventional immunofluorescence microscope (IX71; Olympus, Tokyo, Japan).

\section{Statistical Analysis}

Data are presented as mean \pm s.d. values. All statistical analyses were performed using the MannWhitney $U$-test with the SPSS software (version 14.0; SPSS Inc., Chicago, IL, USA). A probability of $P<0.05$ was considered to be statistically significant.

\section{Results}

\section{Confirmation of Histological Diagnosis in IgG4- Related Disease}

We confirmed that the tissue specimens from all nine cases of submandibular gland IgG4-related disease showed typical histological features of IgG4-related disease, such as lymphoplasmacytic infiltration and dense fibrosis. Additionally, in all cases, numerous IgG4-positive cells were observed and the IgG4-positive/IgG-positive cell ratio was $>40 \%$.

\section{Histological Findings of Sialolithiasis}

The specimens showed lymphoid follicle formation and moderate to severe infiltration of lymphocytes and plasma cells as well as various numbers of neutrophils with various degree of fibrosis. Some specimens included salivary calculus (Figure 1a).

\section{Elevated Expression of Cytokines in IgG4-Related Disease}

The expression of T helper 2 cell cytokines (IL4 and IL5) and regulatory T-cell cytokines (IL10 and TGF $\beta 1$ ) was examined in samples from the nine cases of IgG4-related disease, five cases of sialolithiasis, and six cases of normal submandibular gland. The expression of the IL4, IL5, IL10, and TGF $\beta 1$ cytokines and the $\beta$-actin control in these samples was quantitatively analyzed by real-time PCR. As shown in Figure 1, IgG4-related disease exhibited significantly higher expression ratios of IL4/ $\beta$-actin (31.9 \pm 12.1 -fold higher), IL10/ $\beta$-actin (21.0 \pm 15.7 -fold higher), and TGF $\beta 1 / \beta$-actin (28.6 \pm 23.3-fold higher) than sialolithiasis and normal submandibular gland $(P<0.05)$. In contrast, no significant difference was observed between the IL5/ $\beta$-actin ratio in IgG4-related disease $(0.606 \pm$ $1.13)$ and those in sialolithiasis $(0.119 \pm 0.07)$ and normal submandibular gland $(0.462 \pm 0.369)$ (Figure 1b).

Next, the real-time PCR results were supported via immunostaining of cells by using primary antibodies against IL4, IL5, IL10, and TGF $\beta 1$ (Figure 2a).
The number of IL4-positive cells was significantly higher in IgG4-related disease (4.04 \pm 3.12 cells $/ \mathrm{mm}^{2}$ ) than in submandibular sialolithiasis $\left(0.136 \pm 0.186\right.$ cells $\left./ \mathrm{mm}^{2} ; P<0.01\right)$ and the normal submandibular gland $\left(0.230 \pm 0.356\right.$ cells $/ \mathrm{mm}^{2}$; $P<0.01$ ) (Figure 2b). Similarly, many IL10-positive cells were observed in IgG4-related disease (3.40 \pm 1.84 cells $/ \mathrm{mm}^{2}$ ), whereas the submandibular sialolithiasis and normal submandibular gland contained few IL10-positive cells $\left(0.342 \pm 0.484\right.$ cells $/ \mathrm{mm}^{2}$, $P<0.01 ; 0.226 \pm 0.277$ cells $/ \mathrm{mm}^{2}, P<0.01$, respectively) (Figure 2b). TGF $\beta 1$-positive cells were also more abundant in IgG4-related disease (4.29 \pm $2.37 \mathrm{cells} / \mathrm{mm}^{2}$ ) than in the submandibular sialolithiasis $\left(1.51 \pm 1.11\right.$ cells $\left./ \mathrm{mm}^{2} ; P<0.05\right)$ and normal submandibular gland $\left(0.626 \pm 0.548 \mathrm{cells} / \mathrm{mm}^{2}\right.$; $P<0.01$ ) (Figure 2b). Furthermore, we observed that TGF $\beta 1$-positive cells tended to infiltrate fibrous lesions.

In IgG4-related disease and control groups, the number of IL5-positive cells was much less than that of the other cytokines examined. No significant differences were observed between the number of IL5positive cells in IgG4-related disease tissue (1.58 \pm $\left.1.44 \mathrm{cells} / \mathrm{mm}^{2}\right)$, sialolithiasis $(0.838 \pm 0.531$ cells/ $\left.\mathrm{mm}^{2} ; P=0.450\right)$, and normal submandibular gland tissue $\left(0.811 \pm 0.290\right.$ cells $\left./ \mathrm{mm}^{2} ; P=0.332\right)$ (Figure $\left.2 b\right)$.

\section{Increased Density and Cytokine-Related Distribution of Mast Cells in IgG4-Related Disease}

We compared the number of mast cells in the IgG4related disease and control groups via immunostaining by using an antibody for KIT, which is a marker for mast cells. The number of KIT-positive mast cells was higher in IgG4-related disease $(72.2 \pm 24.5$ cells/ $\mathrm{mm}^{2}$ ) than in the normal submandibular gland $\left(30.0 \pm 11.9\right.$ cells $\left./ \mathrm{mm}^{2} ; P<0.01\right)$ (Figure 3$)$. However, no significant difference was observed between the number of mast cells in the IgG4-related disease and the submandibular sialolithiasis $(177 \pm 269$ cells/ $\mathrm{mm}^{2} ; P=0.73$ ) (Figure 3). Interestingly, the morphological features and distribution of the mast cells were similar to those of the T helper 2 (IL4 and IL5) or regulatory T-cell (IL10 and TGF $\beta 1$ ) cytokinepositive cells. Furthermore, dual immunofluorescence assays showed that KIT-positive mast cells were also positive for each of the IL4, IL5, IL10, and TGF $\beta 1$ cytokines (Figure 4). Additionally, although only a small number of IL5-positive cells was detected in the immunohistochemical experiments, these cells also exhibited KIT coexpression (Figure 4).

\section{T-Cell Distribution in IgG4-Related Disease and Control Groups}

To assess the number and distribution of T cells, we performed immunostaining assays with an antibody against FOXP3, which is a regulatory T-cell marker. 
a

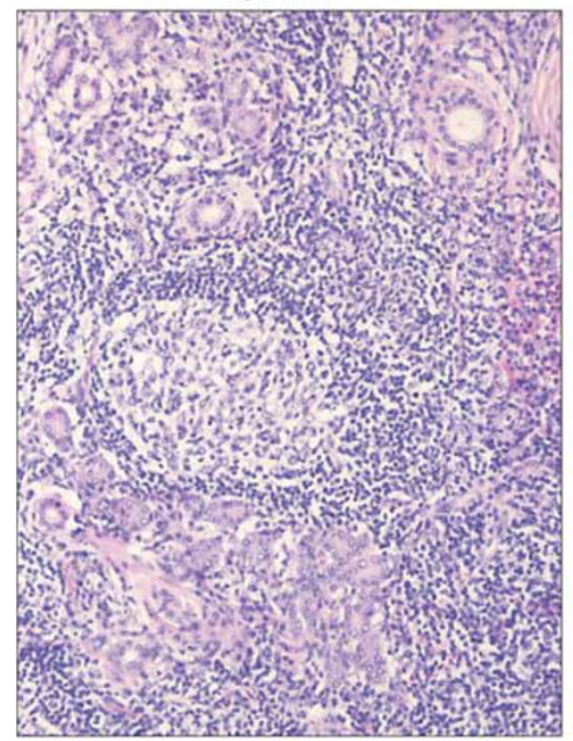

Sialolithiasis

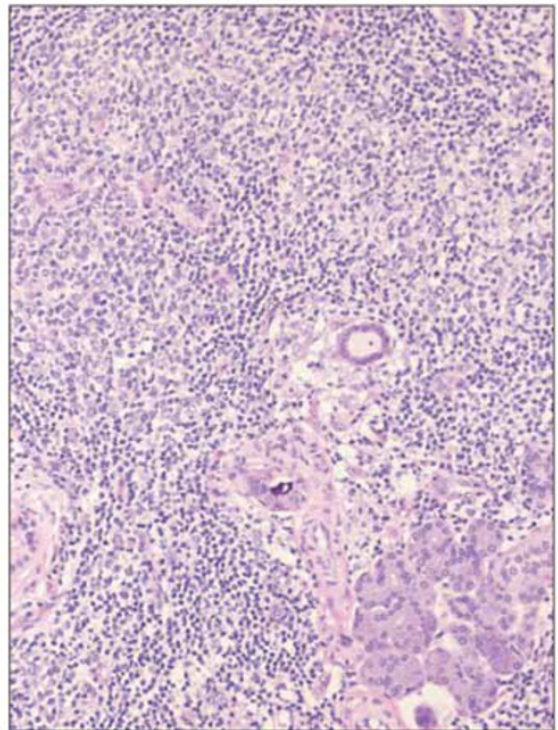

b
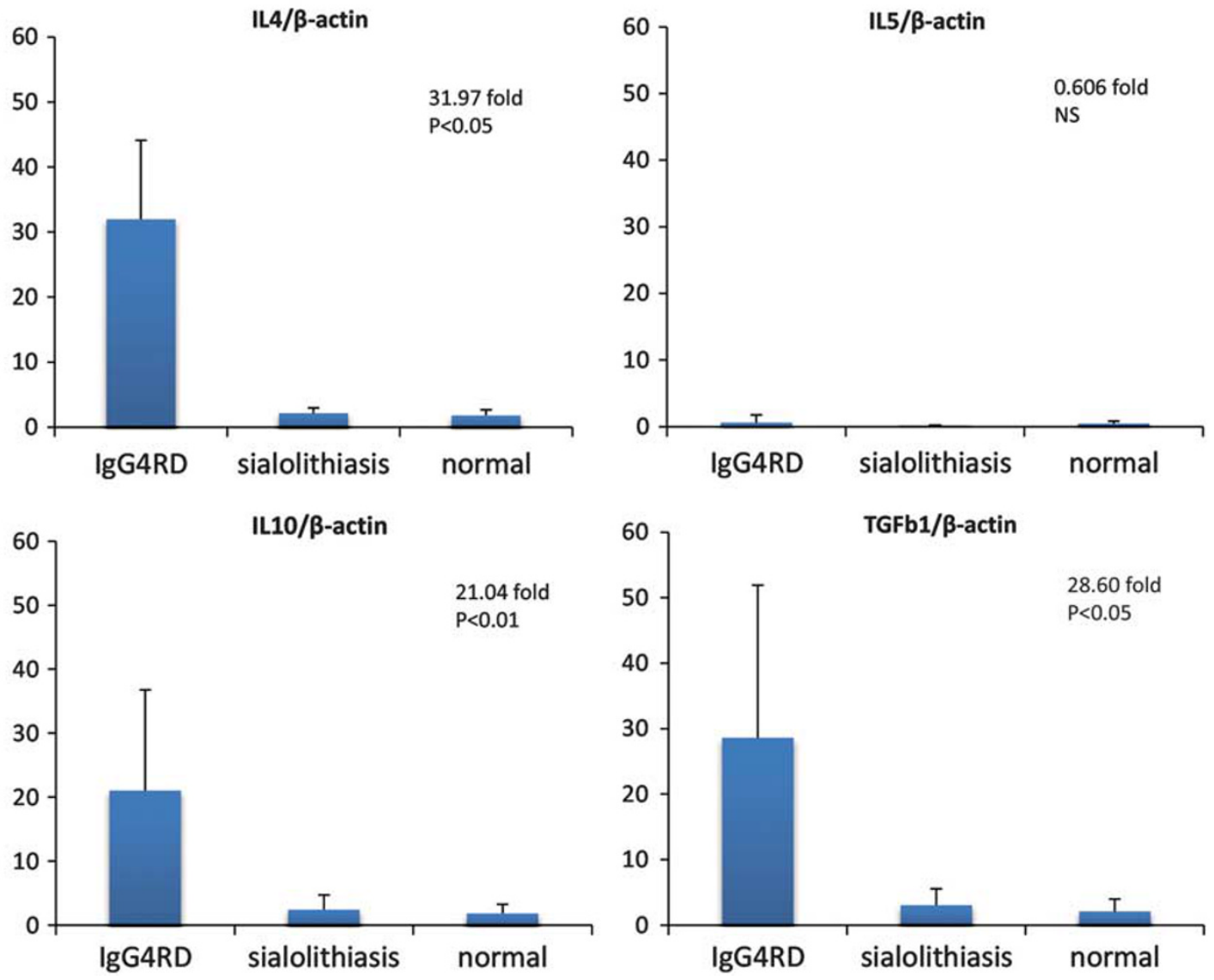

Figure 1 Histological findings and real-time PCR analysis of cytokine expression in IgG4-related disease and control groups. (a) IgG4related submandibular disease showed dense lymphoplasmacytic infiltration with lymphoid follicle (hematoxylin and eosin, H\&E, left). Sialolithiasis revealed moderate to severe lymphoplasmacytic infiltration with lymphoid follicle and calculus (H\&E, center). Normal submandibular gland (H\&E, right). (b) The histograms show the relative quantity of mRNA of the cytokines. The expression ratio of interleukin 4 (IL4)/ $\beta$-actin, IL10/ $\beta$-actin, and transforming growth factor beta 1 (TGF $\beta 1$ (/ $\beta$-actin were significantly higher in IgG4-related submandibular gland disease than in submandibular sialolithiasis and normal submandibular gland. The expression ratio of IL5/ $\beta$-actin was low in all tissues and was not significantly different between the IgG4-related disease and control groups. (IgG4RD, submandibular IgG4-related disease; sialolithiasis, submandibular sialolithiasis; normal; normal submandibular gland). 

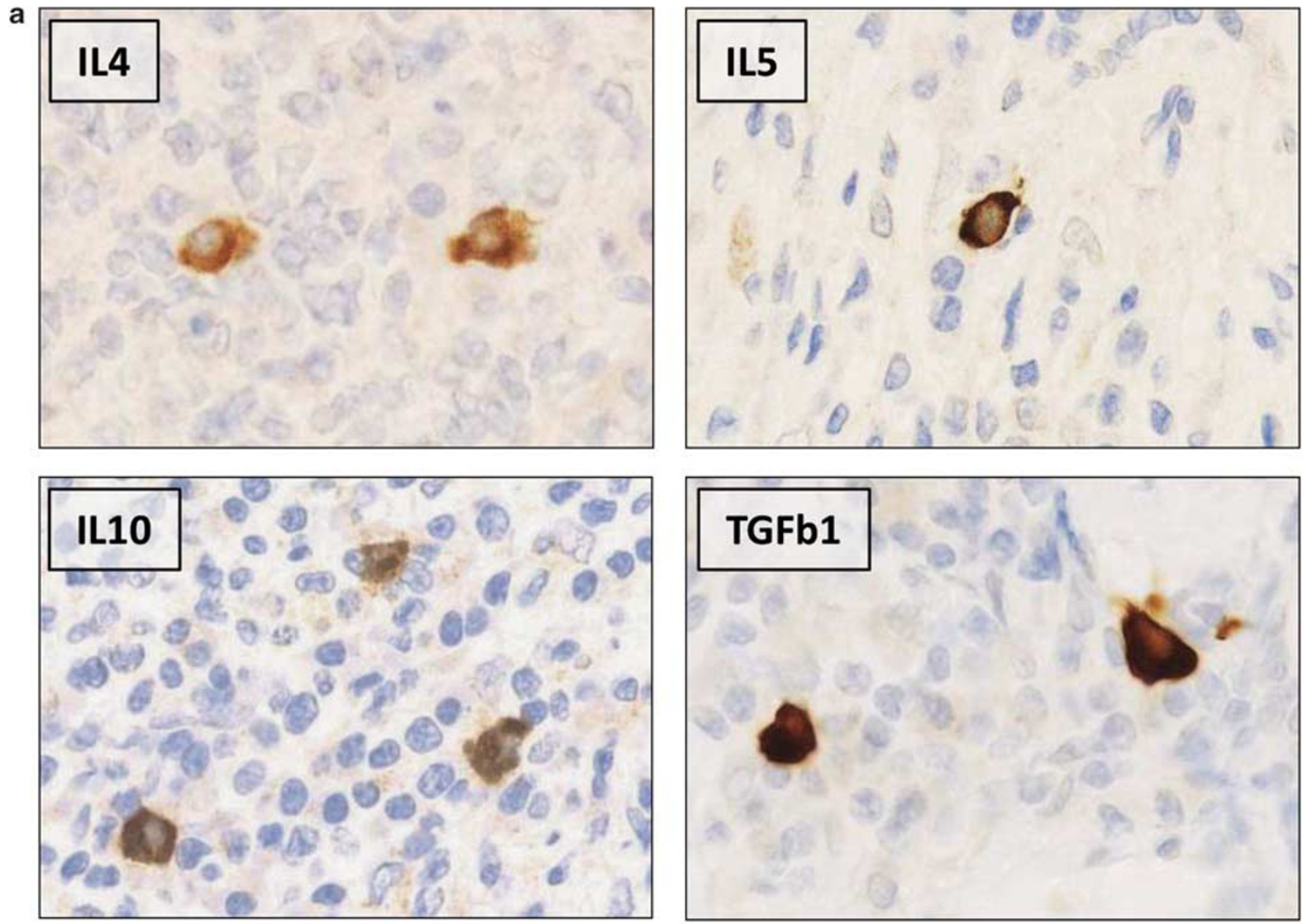

b
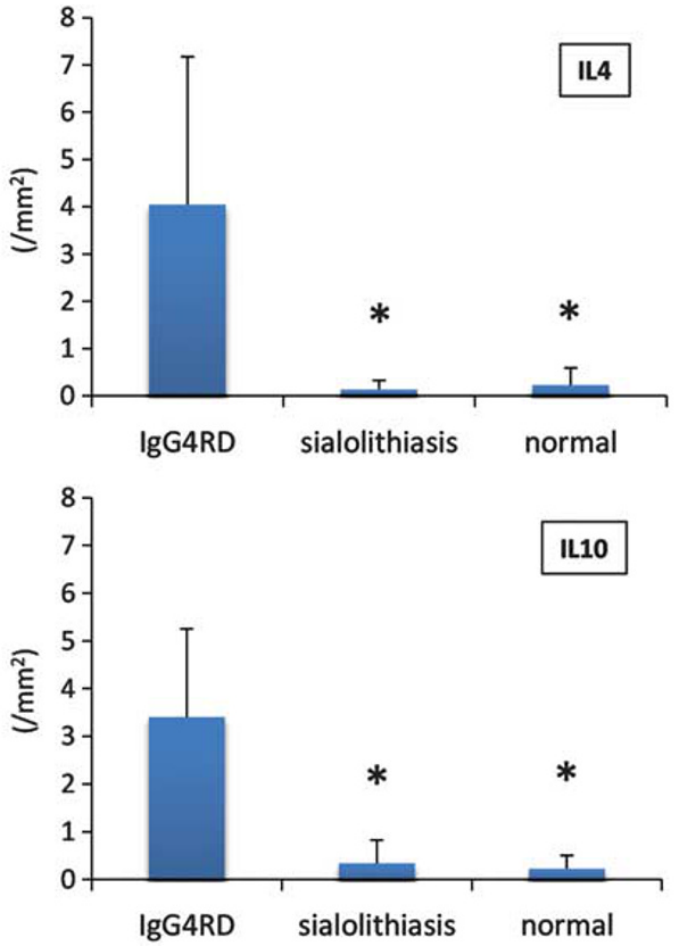
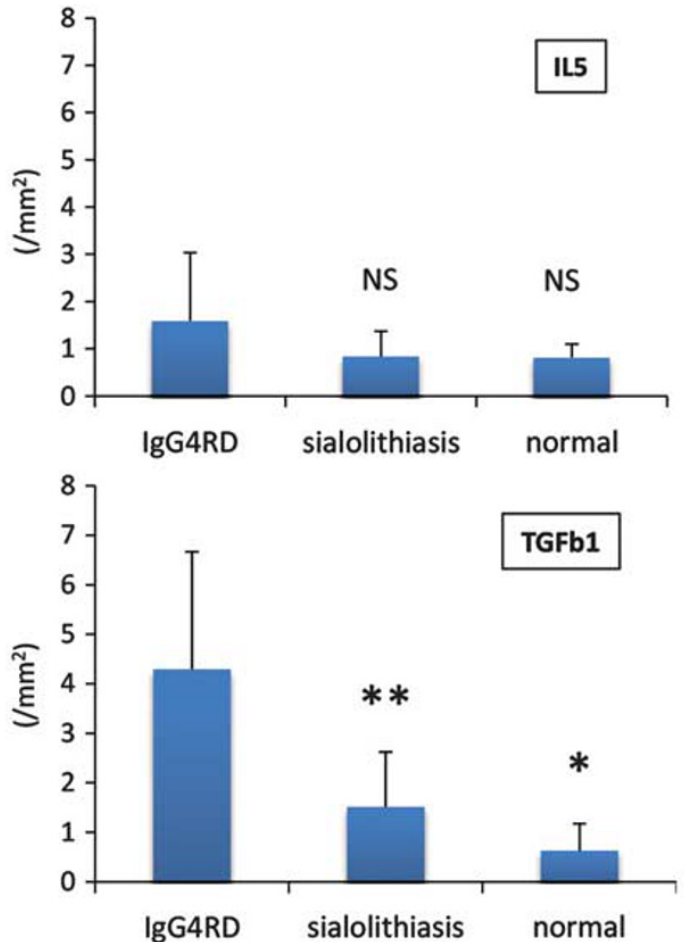

Figure 2 Immunohistochemical analysis of cytokine-expressing cells in IgG4-related disease and control groups. (a) Cells stained with antibodies against the indicated cytokines. (b) The number of cells positive for interleukin 4 (IL4), IL10, and transforming growth factor beta 1 (TGF $\beta 1$ ) were counted per $\mathrm{mm}^{2}$ and significantly larger in IgG4-related disease than in the control groups. Consistent with the results of real-time PCR, the number of IL5-positive cells in IgG4-related disease was lesser than those of other cytokines, and no significant difference was observed between the IgG4-related disease and control groups $\left({ }^{*} P<0.01,{ }^{*} P<0.05\right.$ ). (IgG4RD, submandibular IgG4-related disease; sialolithiasis, submandibular sialolithiasis; normal; normal submandibular gland). 
The number of FOXP3-positive lymphocytes was significantly greater in the submandibular gland IgG4-related disease $\left(834 \pm 284\right.$ cells $\left./ \mathrm{mm}^{2}\right)$ than in submandibular sialolithiasis (435 \pm 330 cells $/ \mathrm{mm}^{2}$; $P<0.05)$ and normal submandibular gland (51.2 \pm 33.5 cells $/ \mathrm{mm}^{2} ; P<0.01$ ) (Figure 5). However, the distribution of FOXP3-positive cells was different from that of cytokine-positive cells, and no FOXP3/ cytokine double-positive cells were observed in dual immunostaining assays (Figure 6). Additionally, we observed that the distribution of CD4-positive lymphocytes was similar to that of FOXP3-positive

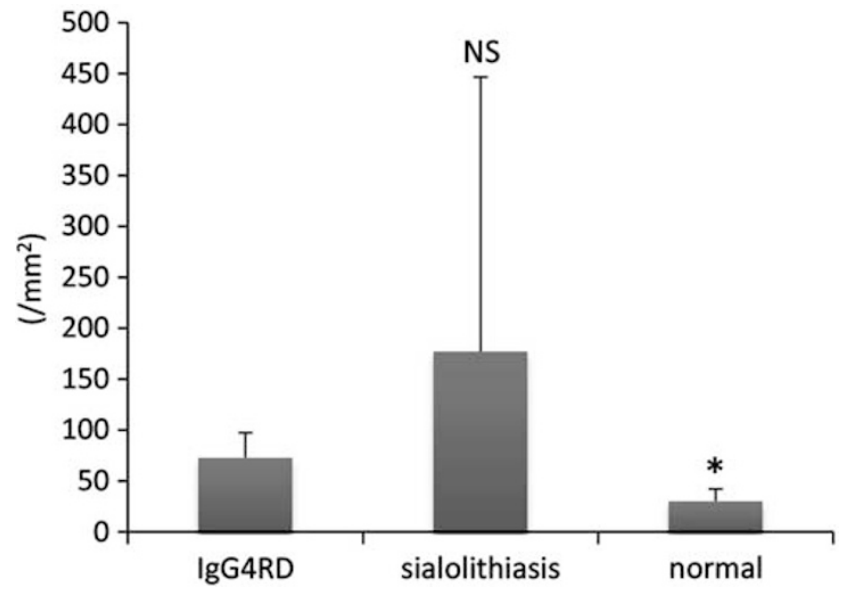

Figure 3 The number of KIT-positive mast cells in IgG4-related disease and control groups. Positive cells were counted per $\mathrm{mm}^{2}$. ( ${ }^{*}<<0.01$; IgG4RD, submandibular IgG4-related disease; sialolithiasis, submandibular sialolithiasis; normal; normal submandibular gland).

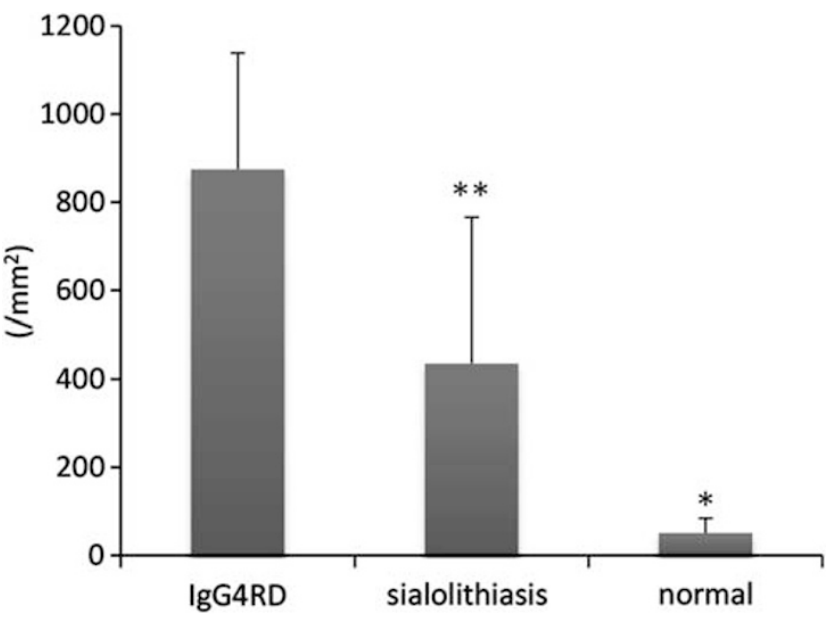

Figure 5 The number of FOXP3-positive lymphocytes. Positive cells were counted per $\mathrm{mm}^{2} .\left({ }^{\star} P<0.01,{ }^{*} P<0.05\right.$; gG4RD, submandibular IgG4-related disease; sialolithiasis, submandibular sialolithiasis; normal; normal submandibular gland).
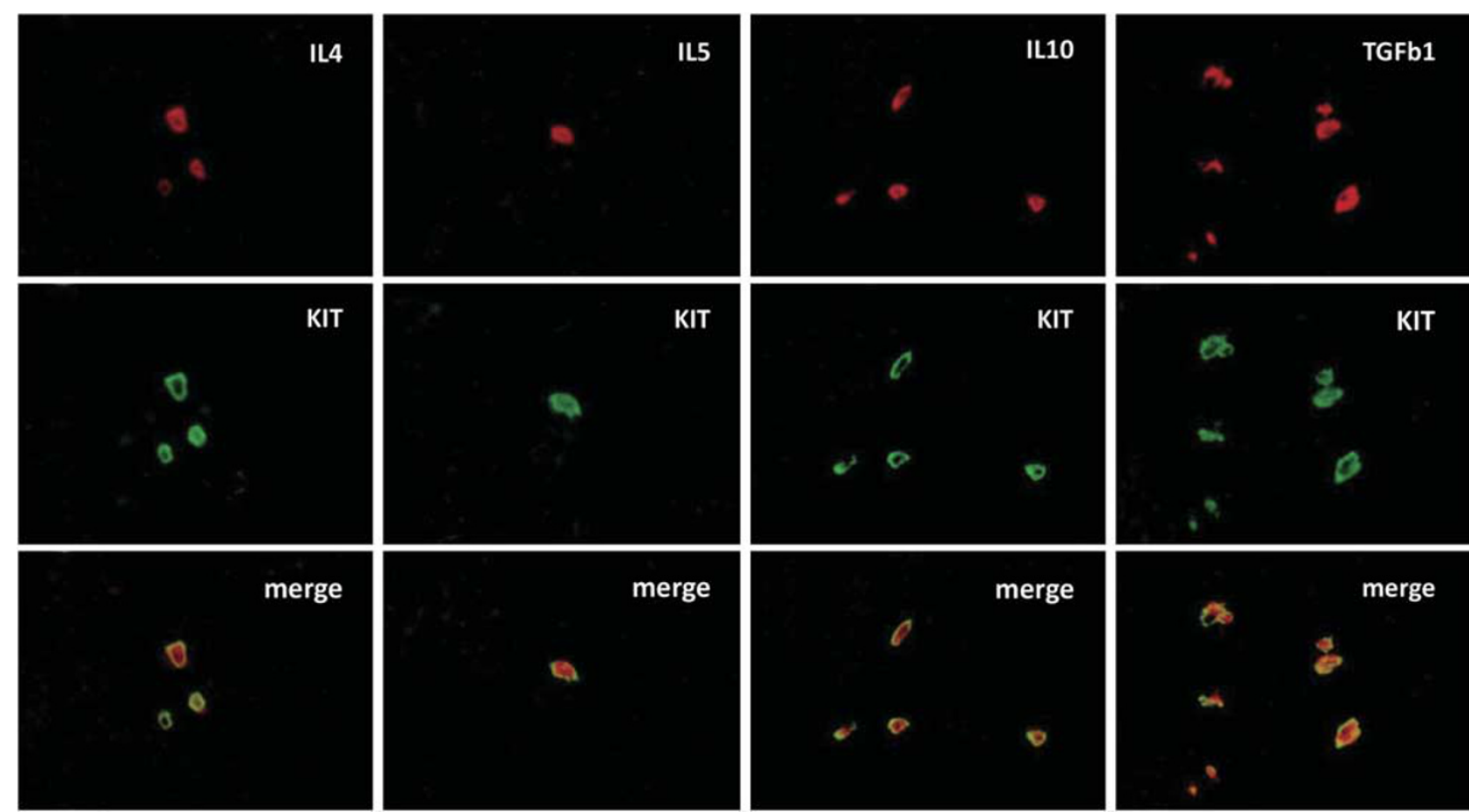

Figure 4 Dual fluorescent analysis of KIT-positive mast cells and cytokine-positive cells in IgG4-related submandibular gland disease. Compared with the results for normal submandibular gland, the number of KIT-positive cells (middle row) was significantly higher in IgG4-related disease and sialolithiasis, and there was no significant difference between these two groups in this regard. Immunostaining for each cytokine (top row) revealed strong cytoplasmic positivity. Positive cells were morphologically similar to mast cells. Dual fluorescent immunostaining detected many positive cells for each cytokine and KIT. The merged image (bottom row) demonstrated double-positive cells for KIT and interleukin 4 (IL4), IL5, IL10, and TGF $\beta 1$. 


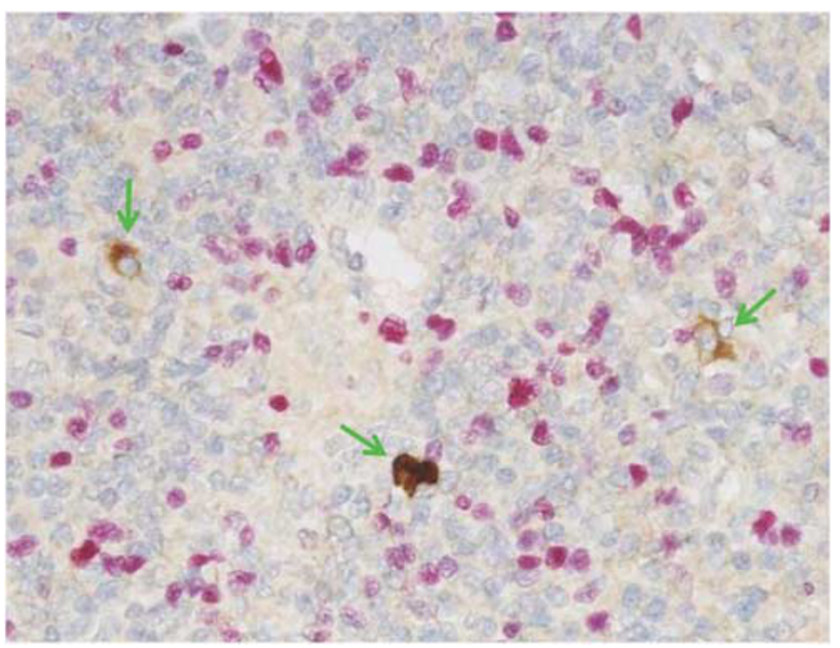

Figure 6 Dual immunostaining of transforming growth factor beta 1 (TGF $\beta 1$ ) and FOXP3. The distribution of TGF $\beta 1$ - and FOXP3positive cells were different (TGF $\beta 1$ /brown, FOXP3/red).

cells, and CD4-positive cell distribution did not correlate with cytokine-positive cell distribution. Moreover, no double-positive cells were observed upon double-immunostaining for each of the cytokines with CD4.

\section{Staining Pattern of IgE in Mast Cells of IgG4-Related Disease}

In IgG4-related disease and control groups tested, a high number of mast cells were positive for IgE with varying density and intensity. As is typical for IgE immunostaining, IgE was observed to be weakly-tomoderately expressed on cell surface membranes (Figure 7a). No significant differences were observed in the number of surface membrane IgE-positive cells among the IgG4-related disease and control groups. However, the IgG4-related disease contained a large number of mast cells that were strongly positive for cytoplasmic IgE (Figure 7a). The distribution of the strongly cytoplasmic IgE-positive cells correlated with KIT expression, and the membranous colocalization of IgE and KIT was confirmed by dual immunofluorescence (Figure 7b).

The number of strongly cytoplasmic IgE-positive cells was significantly greater in IgG4-related disease $\left(7.19 \pm 5.11 \mathrm{cells} / \mathrm{mm}^{2}\right)$ than in sialolithiasis $\left(0.068 \pm 0.15\right.$ cells $\left./ \mathrm{mm}^{2} ; \quad P<0.01\right)$ and normal $\left(0.45 \pm 0.51\right.$ cells $\left./ \mathrm{mm}^{2} ; \quad P<0.01\right) \quad$ control tissues (Figure 7c).

\section{Discussion}

In this study, we confirmed the upregulation of $\mathrm{T}$ helper 2 (IL4) and regulatory T-cell (IL10 and TGF $\beta 1$ ) cytokines in the paraffin-embedded tissue of the submandibular gland IgG4-related disease by real-time PCR. Upregulation of IL5 was not observed in this study, which is similar to previously published data on real-time PCR for paraffin-embedded tissue samples for IgG4-related disease. ${ }^{11}$ Interestingly, our immunohistochemical studies detected a large number of mast cells expressing T helper 2 (IL4) and regulatory T-cell (IL10 and TGF $\beta 1$ ) cytokines in the submandibular gland IgG4-related disease, whereas this was not the case for the control groups. Notably, although the overall number of mast cells was similar among the samples of submandibular gland IgG4-related disease, submandibular sialolithiasis, and normal submandibular gland, the IgG4-related disease contained significantly more cytokineexpressing mast cells. Furthermore, interestingly, our immunohistochemical experiments provided no evidence that $\mathrm{T}$ cells were producing the upregulated cytokines. In our study, we considered FOXP3positive cells to be regulatory T cells, as FOXP3 is considered to be a specific marker of regulatory $\mathrm{T}$ cells, although a minor amount of effector $\mathrm{T}$ cells can exhibit transient expression of FOXP3. ${ }^{12-14}$ It has been suggested that $\mathrm{T}$ helper 2 and regulatory $\mathrm{T}$ cells are most important in the pathogenesis of IgG4-related disease. Although we did find that the number of FOXP3-positive regulatory T cells was significantly increased in IgG4-related disease in our study, we did not find any evidence that the FOXP3positive regulatory $\mathrm{T}$ cells produced regulatory cytokines. Thus, our results do not support the hypothesis that $\mathrm{T}$ cells express the cytokines associated with IgG4-related disease; rather, our data indicate that mast cells are the source of these upregulated cytokines. However, a close relationship between regulatory $\mathrm{T}$ cells and mast cells has been reported, and TGF $\beta 1$ has been shown to promote differentiation of naive $\mathrm{T}$ cells to regulatory $\mathrm{T}$ cells. ${ }^{15}$ Therefore, we hypothesized that the upregulated mast cells produce TGF $\beta 1$, which secondarily promotes the differentiation of naïve $\mathrm{T}$ cells to regulatory $\mathrm{T}$ cells in the tissue affected.

We observed that mast cells in IgG4-related disease were strongly positive for IgE. This result suggests that IgE is associated with the pathogenesis of IgG4-related disease. Consistent with this notion, patients with IgG4-related disease frequently have an allergic background, such as allergic rhinitis, bronchial asthma, and atopic dermatitis. In addition, patients with this disease typically show elevated serum IgE levels as well as elevated IgG4 levels. ${ }^{16} \mathrm{IgE}$ is a key stimulator of mast cells, and the mechanism underlying IgE stimulation of mast cells to induce various immunological cascades has been extensively studied. Notably, chronic elevation of antigen-independent, non-specific IgE upregulates the high-affinity IgE receptor, FceRI, on mast cells, which inhibits mast cell apoptosis and promotes cytokine production. ${ }^{17}$ Our observation that mast cells in IgG4-related disease were strongly positive for IgE supports the idea that IgE-mediated stimulation of mast cells may have a role in the 
a

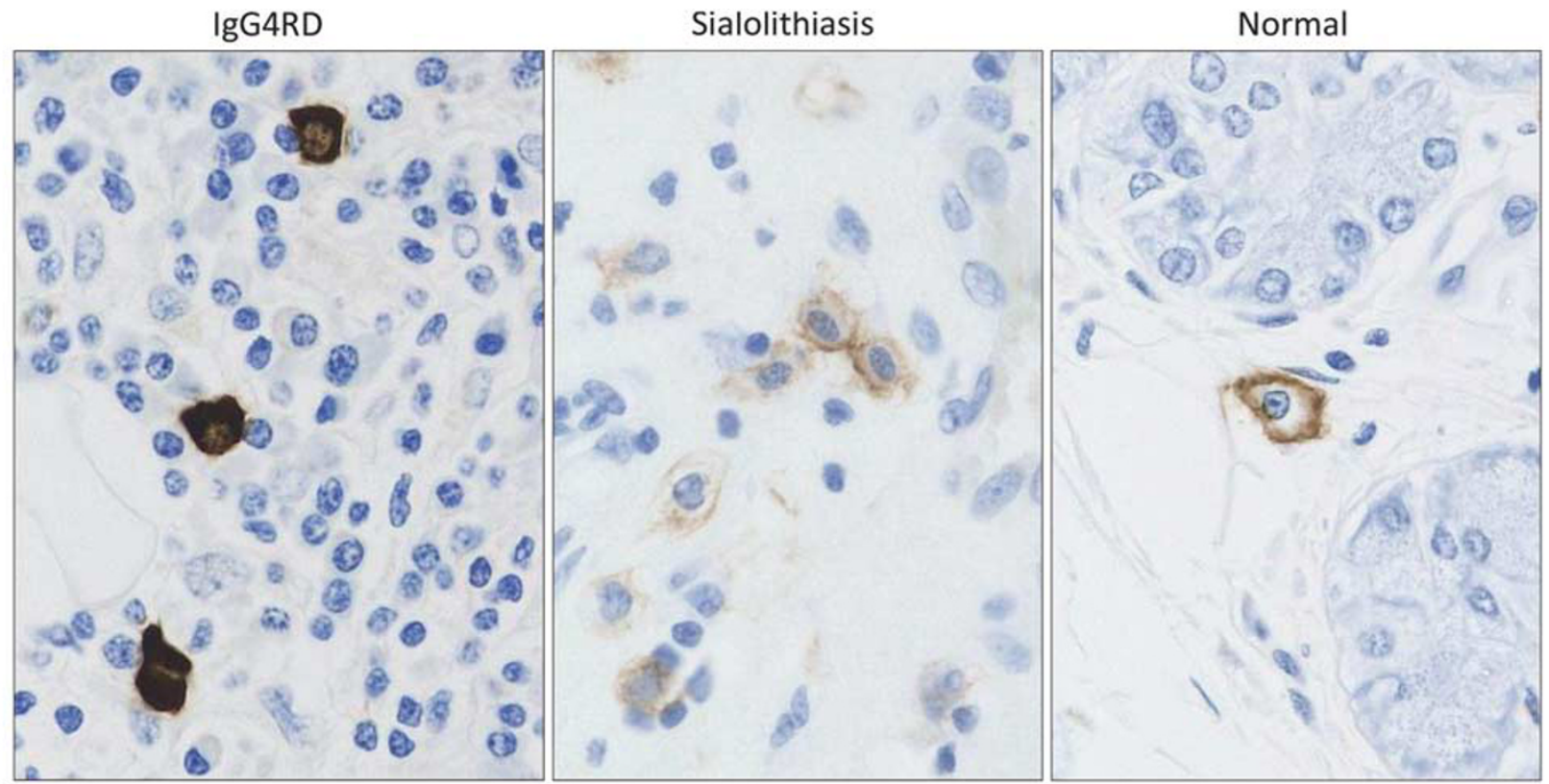

b
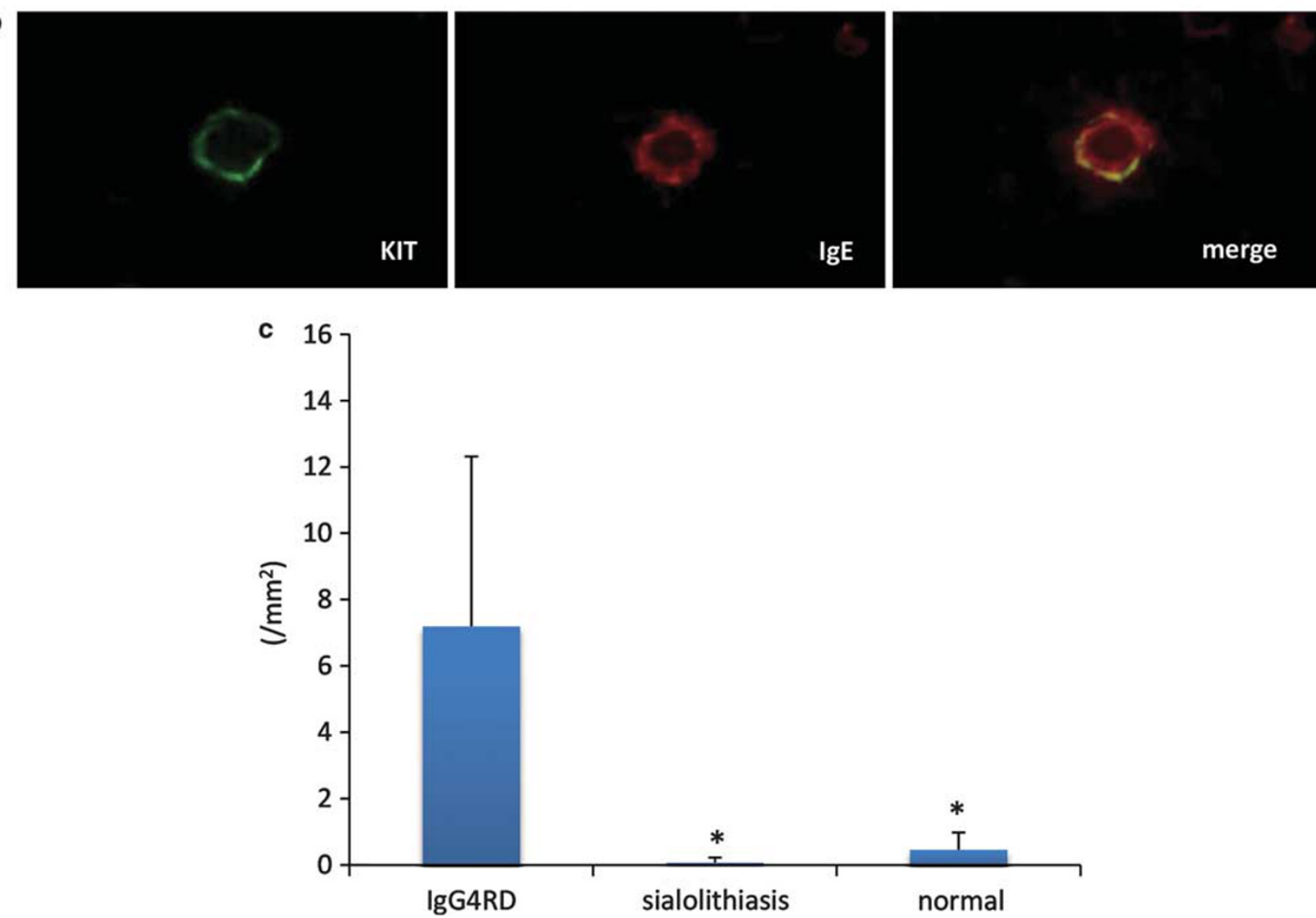

Figure 7 IgE expression in mast cells. (a) In IgG4-related disease, mast cells showed strong cytoplasmic positivity for IgE. In contrast, mast cells in sialolithiasis and normal submandibular gland showed only moderate-to-weak IgE positivity and membranous localization. (b) Colocalization of IgE- and KIT-positive cells was confirmed by dual immunofluorescence (IgE/red, KIT/green). (c) The number of strongly cytoplasmic IgE-positive cells was counted per $\mathrm{mm}^{2}$ and was significantly different between the IgG4-related disease and control groups. ( $\left.{ }^{*} P<0.01\right)$ (IgG4RD, submandibular IgG4-related disease; sialolithiasis, submandibular sialolithiasis; normal; normal submandibular gland). 
pathogenesis of IgG4-related disease. However, it remains to be determined why the IgE in these cells was localized to the cytoplasm. Previous studies have shown that the number of $\mathrm{Fc}_{\mathrm{C}} \mathrm{RI}$ receptors on mast cells greatly increases in the presence of elevated serum IgE levels. ${ }^{18}$ Thus, it is possible that what appeared to be cytoplasmic localization of IgE in our immunostaining experiments may have actually been the result of very high levels of membrane-associated IgE. However, a few reports have suggested that antigen cross-linking could lead to the aggregation and internalization of FceRI. In particular, IgE was detected in the cytoplasm of mast cells as a result of the internalization and endocytosis of FceRI. ${ }^{19}$ These data suggest that the continuous upregulation of mast cells might result in endocytosis-mediated cytoplasmic accumulation of IgE. However, this hypothesis should be based on the existence of certain antigens, and the role of internalization of FceRI remains controversial.

Steroid therapy has been the first choice of medication for IgG4-related disease. However, a recent study reported a case of IgG4-related disease that regressed after treatment with an antihistamine agent (epinastine hydrochloride) alone, which indicates an allergic background to the disease and may indirectly suggest the involvement of mast cells. ${ }^{20}$ The in vitro effects of antihistamines on the inhibition of mediator release from mast cells have been reported. ${ }^{21}$ Moreover, an in vitro study of human conjunctival mast cells revealed that epinastine inhibited mast cell secretion of cytokines, including IL10. ${ }^{22}$ However, it remains unclear why antihistamines inhibit cytokine secretion, although H1 antagonism seems unrelated.
Rituximab is another treatment currently being used for refractory cases of IgG4-related disease. Rituximab therapy has been reported to produce rapid regression in refractory patients. ${ }^{23}$ Additionally, rituximab therapy was found to induce regression of symptoms with a decrease in serum IgE levels, ${ }^{24}$ and the concentration of $\operatorname{IgE}$ showed a steeper decline than serum IgG4 levels. These results provide additional evidence that the allergic reaction mediated by $\operatorname{IgE}$ is an important factor in the regulation of IgG4-related disease.

Hyper-IgE syndrome is a complex immunodeficiency characterized by atopic dermatitis associated with extremely high serum IgE levels and susceptibility to infections with extracellular bacteria. ${ }^{25}$ Despite the continuously elevated serum IgE level, hyper-IgE syndrome shows clinical features that are quite different from those of IgG4-related disease. Furthermore, hyper-IgE syndrome has been linked to mutations in signal transducer and activator of transcription 3 (STAT3) and tyrosine kinase 2 (TYK2), and to defective signal transduction pathways involving multiple cytokines. In contrast to this downregulation of cytokine signaling, IgG4-related disease is associated with the upregulation of multiple cytokine signals.

To our knowledge, this study provides the first evidence relating mast cells to IgG4-related disease. However, as mast cells are currently known as powerful mediators of the immune response and are closely related with allergic responses, the involvement of mast cells in IgG4-related disease seems logical. Moreover, mast cells have been implicated in other inflammatory diseases. For instance, it was reported that a large number of

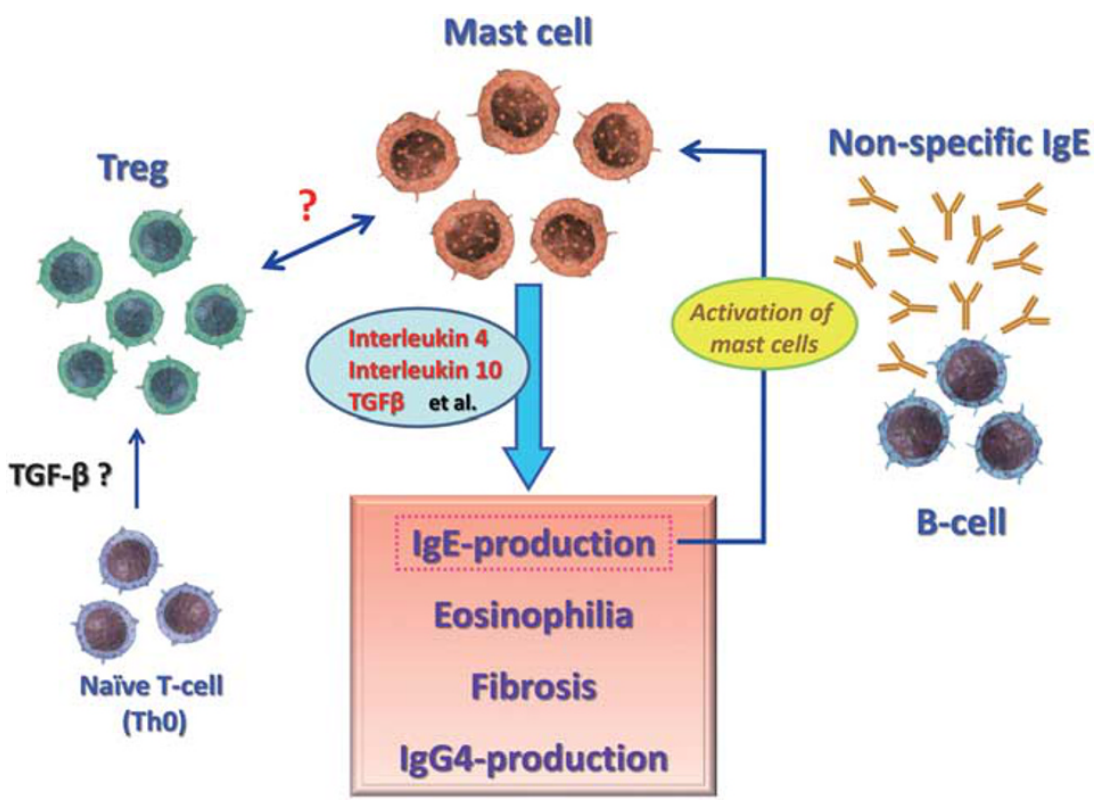

Figure 8 Model for the pathogenesis of IgG4-related disease. Because of an allergic background with the elevation of non-specific IgE levels, activated mast cells produce interleukin 4 (IL4), IL10, and transforming growth factor beta 1 (TGF $\beta 1$ ), which induce the distinctive features of IgG4-related disease. In addition, IL4 and IL10 themselves induce IgE production to upregulate mast cells, and TGF $\beta 1$ induces the transformation of naive $\mathrm{T}$ cells to regulatory $\mathrm{T}$ cells. The relationship between regulatory $\mathrm{T}$ cells and mast cells remains unclear. 
mast cells was observed in periductal and ductal fibrosis in primary sclerosing cholangitis (PSC) and chronic sclerosing sialadenitis, indicating that mast cells might contribute to fibrosis. ${ }^{26,27}$ Mast cell expression of IL10, as observed in our immunohistochemical experiments, may affect mast cell involvement in IgG4-related disease. IL10 is known to be an anti-inflammatory cytokine that can suppress acquired or innate immune responses. Mast cell-derived IL10 has been reported to limit contact dermatitis and chronic irradiation with ultraviolet B. ${ }^{28}$ Similarly, in IgG4-related disease, mast cell production of IL10 may prevent allergic inflammation in the affected organs.

On the basis of results of our study and those of previously published reports, we established a hypothesis for the pathogenesis of IgG4-related disease, which is summarized in Figure 8. Patients with IgG4-related disease usually have an allergic background with elevation of non-specific IgE levels. This antigen-independent non-specific IgE binds to FceRI on mast cells to promote the production of T helper 2 (IL4) and regulatory T-cell (IL10 and TGF $\beta 1$ ) cytokines, which induce the distinctive features of IgG4-related disease such as lymphoplasmacytic infiltration, interstitial fibrosis, and IgG4 production. In addition, IL4 and IL10 induce IgE production, which promotes the upregulation of mast cells. Although it is possible that regulatory $\mathrm{T}$ cells interact with mast cells, their involvement in this process is uncertain and they might be secondarily recruited to the affected tissue. According to this scenario, rituximab might exert its effects through the inhibition of IgE-production of B-cells (Figure 8). This model is based on the major finding of this study, which indicates that mast cells have a key role in IgG4-related disease.

\section{Acknowledgments}

This work was supported by a Grant-in-Aid for Scientific Research (C) (no. 24591447) from the Japan Society for the Promotion of Science and 'Research on Measures for Intractable Disease' Project: matching fund subsidy from Ministry of Health Labour and Welfare, Japan.

\section{Author contributions}

Conceived and designed the experiments: YS. Performed the experiments: MT and YS. Analyzed the data: YS, MT, ST, KO, KT, YG, and TI. Contributed materials: YO and TT. Wrote the paper: MT, YS, and TY. All authors read and approved the final manuscript.

\section{Disclosure/conflict of interest}

The authors declare no conflict of interest.

\section{References}

1 Umehara H, Okazaki K, Masaki Y, et al. A novel clinical entity IgG4-related disease (IgG4RD): general concept and details. Mod Rheumatol 2012;22:1-14.

2 Deshpande V, Zen Y, Chan JK, et al. Consensus statement on the pathology of IgG4-related disease. Mod Pathol 2012;25:1181-1192.

3 Sato Y, Inoue D, Asano N, et al. Association between IgG4-related disease and progressively transformed germinal centers of lymph nodes. Mod Pathol 2012;25:956-967.

4 Sato Y, Takeuchi M, Takata K, et al. Clinicopathologic analysis of IgG4-related skin disease. Mod Pathol 2012;26:523-532.

5 Zen Y, Fujii T, Harada K, et al. Th2 and regulatory immune reactions are increased in immunoglobin G4-related sclerosing pancreatitis and cholangitis. Hepatology 2007;45:1538-1546.

6 Tanaka A, Moriyama M, Nakashima H, et al. Th2 and regulatory immune reactions contribute to IgG4 production and the initiation of Mikulicz disease. Arthritis Rheum 2012;64:254-263.

7 Khosroshahi A, Bloch DB, Deshpande V, et al. Rituximab therapy leads to rapid decline of serum IgG4 levels and prompt clinical improvement in IgG4-related systemic disease. Arthritis Rheum 2010; 62:1755-1762.

8 Gri G, Frossi B, D’Inca F, et al. Mast cell: an emerging partner in immune interaction. Front Immunol 2012;3:120.

9 Amin K. The role of mast cells in allergic inflammation. Respir Med 2012;106:9-14.

10 Kashiwakura J, Kawakami Y, Yuki K, et al. Polyclonal IgE induces mast cell survival and cytokine production. Allergol Int 2009;58:411-419.

11 Nakashima H, Miyake K, Moriyama M, et al. An amplification of IL-10 and TGF-beta in patients with IgG4-related tubulointerstitial nephritis. Clin Nephrol 2010;73:385-391.

12 Fontenot JD, Gavin MA, Rudensky AY. Foxp3 programs the development and function of CD4 + CD25 + regulatory T cells. Nat Immunol 2003;4:330-336.

13 Miyao T, Floess S, Setoguchi R, et al. Plasticity of Foxp3(+) T cells reflects promiscuous Foxp3 expression in conventional $\mathrm{T}$ cells but not reprogramming of regulatory T cells. Immunity 2012;36:262-275.

14 Da Silva Martins M, Piccirillo CA. Functional stability of Foxp3 + regulatory T cells. Trends Mol Med 2012; 18:454-462.

15 Zhang $\mathrm{W}, \mathrm{Wu} \mathrm{K}, \mathrm{He} \mathrm{W}$, et al. Transforming growth factor beta 1 plays an important role in inducing $\mathrm{CD} 4(+)$ CD25( + ) forhrad box P3(+ ) regulatory t cells by mast cells. Clin Exp Immunol 2010;161: 490-496.

16 Sato Y, Notohara K, Kojima M, et al. IgG4-related disease: historical overview and pathology of hematological disorders. Pathol Int 2010;60:247-258.

17 Bax HJ, Keeble AH, Gould HJ. Cytokinergic IgE action in mast cell activation. Front Immunol 2012;3:229.

18 Burton OT, Oettgen HC. Beyond immediate hypersensitivity: evolving roles for IgE antibodies in immune homeostasis and allergic diseases. Immunol Rev 2011;242:128-143.

19 Fattakhova GV, Masilamani M, Nrayanan S, et al. Endosomal trafficking of the ligated FCeRI receptor. Mol Immunol 2009;46:793-802. 
20 Ohshima K, Sato Y, Yoshino T. A case of IgG4-related dacryoadenitis regressed without systemic steroid administration. J Clin Exp Hematol 2013;53:53-56.

21 Cuss FM. Beyond the histamine receptor: effect of antihistamines on mast cells. Clin Exp Allergy 1999; 29:4-59.

22 Galatowicz G, Ajayi Y, Stern ME, et al. Ocular antiallergic compounds selectively inhibit human mast cell cytokines in vitro and conjunctival cell infiltration in vivo. Clin Exp Allergy 2007;37:1648-1656.

23 Khosroshahi A, Carruthers MN, Deshpande V, et al. Rituximab for the treatment of IgG4-related disease: lessons from 10 consecutive patients. Med Baltimore 2012;91:57-66.

24 Wong PC, Fung AT, Gerrie AS, et al. IgG4-related disease with hypergammaglobulinemic hyperviscosity and retinopathy. Eur J Haematol 2012;90:250-256.
25 Minegishi Y. Hyper-IgE syndrome. Curr Opin Immunol 2009;21:487-492.

26 Tsuneyama K, Kono N, Yamashiro M, et al. Aberrant expression of stem cell factor on biliary epithelial cells and peribiliary infiltration of c-kit-expressing mast cells in hepatolithiasis and primary sclerosing cholangitis: a possible contribution to bile duct fibrosis. J Pathol 1999;189:609-614.

27 Tsuneyama K, Saito K, Ruebner BH, et al. Immunological similarities between primary sclerosing cholangitis and chronic sclerosing sialadenitis. Dig Dis Sci 2000;45:366-372.

28 Grimbaldeston MA, Nakae S, Kalesnikoff J, et al. Mast cell-derived interleukin 10 limits skin pathology in contact dermatitis and chronic irradiation with ultraviolet. B Nat Immunol 2007;8: 1095-1104. 